\title{
EPIDEMIOLOGÍA DE LOS TRASTORNOS MENTALES EN CHILE Y SU IMPACTO EN LAS POLÍTICAS PÚBLICAS DE SALUD MENTAL, 1950-1973
}

\author{
Claudia Araya Ibacache \\ Universidad Católica de Chile, PEMHUC \\ Email: c.arayaibacache@gmail.com \\ ORCID iD: https://orcid.org/0000-0001-7353-0123 \\ Camila Martínez Villavicencio \\ Universidad Católica de Chile, PEMHUC \\ Email: cpmartinez2@uc.cl \\ ORCID iD: https://orcid.org/0000-0001-8789-9976
}

Recibido: 4 abril 2020; Aceptado: 22 junio 2020

Cómo citar este artículo/Citation: Araya Ibacache, Claudia; Martínez Villavicencio, Camila (2021) "Epidemiología de los trastornos mentales en Chile y su impacto en las políticas públicas de salud mental, 1950-1973", Asclepio, 73(1): p349. https://doi.org/10.3989/ asclepio.2021.15

RESUMEN: A partir de la década de 1950, un grupo de psiquiatras chilenos comienza a converger en torno a la epidemiología de los trastornos mentales. Ni la psiquiatría ni la salud mental habían logrado posicionarse exitosamente en el escenario público, como sí lo habían conseguido la salud "física" y la medicina. Desde la década anterior los psiquiatras venían buscando ese posicionamiento, fundamentalmente a través de la introducción y aplicación de las terapias biológicas. Planteamos que a partir de la influencia de las ciencias sociales y del escaso éxito de los tratamientos psiquiátricos, el desarrollo de los estudios epidemiológicos significó una renovación en el papel de la psiquiatría y los psiquiatras en el escenario de la salud pública.

PALABRAS CLAVE: Chile; Salud mental; Políticas públicas; Epidemiología; Psiquiatría comunitaria.

\section{EPIDEMIOLOGY OF MENTAL DISORDERS IN CHILE AND ITS IMPACT ON PUBLIC MENTAL HEALTH POLICIES, 1950-1973}

ABSTRACT: Beginning in the 1950s, a group of chilean psychiatrists began to converge around the epidemiology of mental disorders. Neither psychiatry nor mental health had successfully positioned themselves on the public stage, as "physical" health and medicine had. Since the previous decade, psychiatrists had been looking for this positioning, mainly through the introduction and application of biological therapies. We propose that from the influence of the social sciences and the limited success of psychiatric treatments, the development of epidemiological studies meant a renewal in the role of psychiatry and psychiatrists in the public health scene.

KEY WORDS: Chile; Mental Health; Public Policies; Epidemiology; Community Psychiatry. 
Una epidemiología crítica puede ser un instrumento básico de recuperación ética y de construcción de una politicidad; dos aspectos seriamente menoscabados en medio de un proceso neoliberal cínico y regresivo (Breihl, 2013, p.31)

\section{INTRODUCCIÓN}

Cada país nuestro ha tenido en algún momento de los últimos 35 años un grupo de psiquiatras que apuntó al futuro y fue, casi sin excepciones, interferido y paralizado. Paradójicamente, la psiquiatría latinoamericana vive, mayoritariamente, recordando el futuro (Marconi, 1990, p.427). Estas palabras del psiquiatra chileno Juan Marconi evidencian la fractura que ha caracterizado a la historia de la psiquiatría y de la salud mental latinoamericanas. Para Marconi, en distintos momentos de la historia continental, fundamentalmente a partir de la década de 1950, grupos de psiquiatras, médicos y otros profesionales de la salud, diseñaron programas y proyectos más avanzados que, inevitablemente fueron interferidos políticamente, desmembrados los equipos y paralizados los esfuerzos. De ahí que aluda a esa figura paradójica, diciendo que "nosotros no necesitamos tanto el futuro no pronunciado, sino, que estamos recordando el futuro que nos quitaron" (Marconi, 1990, p.435). Lo que aquí nos hemos propuesto es mostrar uno de esos momentos que debió ser futuro, pero que devino, demasiado pronto, según las palabras de Marconi, en pasado.

Desde hace más de una década se viene hablando de la existencia de una crisis de la salud mental en Chile. Si bien no es un tema de la agenda pública, cada cierto tiempo genera alarma y debate en la población, especialmente cuando se publican las cifras de suicidio o de alcoholismo y drogadicción juvenil ${ }^{1}$. Estas alarmantes cifras tampoco van de la mano con el porcentaje del presupuesto fiscal destinado a salud mental. Esta incoherencia, entre la magnitud del problema y la respuesta del Estado, se explica en parte, por la ausencia de un enfoque epidemiológico que, además de caracterizar la situación, permita abordar el problema. Ante este diagnóstico, las palabras de Marconi se vuelven muy actuales y las preguntas surgen casi automáticamente: ¿Cuál es la relación entre psiquiatría, salud mental y epidemiología?; ¿Cuál ha sido el devenir de esta relación en el tiempo?; ¿Por qué, dada la situación de salud mental en el país, no existe un desarrollo consolidado de una epidemiología en ese sentido?

El concepto de salud mental surge a mediados del siglo pasado. Aun cuando su origen implicaba una superación de la exclusividad que la medicina tenía sobre los procesos de salud y enfermedad, su fuerte vinculación con la psiquiatría ha marcado tanto su desarrollo como las dificultades que han existido en los países para abordarla desde lo público. Por lo mismo, las características que ha asumido el desarrollo de la psiquiatría han influido notoriamente en el devenir de la salud mental. En el caso chileno, la profesionalización de la psiquiatría ha estado marcada por su carácter biologicista en lo disciplinario y manicomial en lo asistencial (Araya, 2018). Si bien desde el último tercio del siglo XIX comienzan a surgir críticas a este modelo caracterizado por el encierro y la cronicidad, en Chile, la asistencia preferentemente manicomial se extendió a lo largo de todo el siglo XX (Mendive, 2004).

A las críticas al aislamiento como herramienta terapéutica, le siguió un movimiento de higiene mental surgido en Estados Unidos en las primeras décadas del siglo XX. Las explicaciones físicas de los fenómenos mentales ya no resultaban suficientes; así fue como psiquiatras y científicos sociales, en paralelo con otros movimientos como el de protección a la infancia y la asistencia social, comenzaron a buscar respuesta a los fenómenos de la mente desde una perspectiva social. En 1909, por iniciativa de Clifford Beers, se fundó en Estados Unidos el National Committee for Mental Hygiene. Los estatutos del Comité insistían en la propuesta de "una prevención a través de la educación del público, la investigación y la divulgación de los conocimientos relativos a todas las formas de trastornos psíquicos" (Castel; Castel; Lovell, 1980, p.43) A estas primeras iniciativas reformistas le siguieron las ideas sobre prevención de Adolf Meyer, también en Estados Unidos (Klappenbach, 1989).

Si bien el movimiento de higiene mental volcó a los médicos a la sociedad y amplió la conceptualización de los trastornos psiquiátricos y su forma de asistirlos, esto no significó relegar la psiquiatría al asilo, sino más bien extender la labor del médico especialista a todo el conjunto social. Será a partir de mediados de siglo pasado que la influencia de las ciencias sociales se asentará permanentemente en las discusiones acerca de las delimitaciones entre psiquiatría y salud mental. Estos debates se articularán asimismo con las discusiones respecto a los modelos epidemiológicos que comienzan a instalarse desde la psiquiatría (Ferrero, 2000).

Las primeras prácticas discursivas sobre registros de enfermedades, de una manera más colectiva que individual se dieron a partir del siglo XVII con el posicionamiento de las nociones de población, estado y 
comunidad (Foucault, 2009). Pero su transformación en una especialidad médica moderna, desde el primer tercio del siglo veinte, estuvo influida fuertemente por el evolucionismo biologicista, el causalismo de la física mecánica y el inductivismo empírico predominante en las ciencias naturales (Barradas, 1998). Constituida en base a la "teoría del germen", esta epidemiología se caracterizó por una marcada negación de lo social y lo cultural, al concebir al ser humano "como una especie biológica, dejándose de lado la diversidad social y cultural que existe dentro de ella" (Urquía, 2019, p.65).

Por su parte, los primeros estudios psiquiátricos a nivel mundial datan de las primeras décadas del siglo pasado y se ocuparon de la parálisis general progresiva. Mattauschek y Pilcz, entre 1912 y 1923, realizaron un seguimiento a 4.000 oficiales del ejército austriaco infectados por sífilis para evidenciar la vinculación de la parálisis general progresiva con esta enfermedad (Williams; Wilkinson; Rawnsley, 2019) Más temprano, a pesar de no ser un estudio epidemiológico, una investigación sociológica puso en cuestión el paradigma de la epidemiología basado en las ciencias naturales. Hacia fines del siglo XIX, Emil Durkheim, analizando las estadísticas de suicidio en Europa, había referido cómo la cultura y el ambiente social pueden modificar la conducta del individuo en una sociedad particular (Durkheim, 1897). Inclusive, cómo ciertos fenómenos podrían llegar a causar "epidemias de locura" o "psicopatología del proceso social". Sostuvo además que la cohesión en la sociedad era un componente importante en la prevención del suicidio.

Sin embargo, su obra, considerada como una contribución decisiva al surgimiento de la sociología, no influyó en la naciente epidemiología psiquiátrica. "El ejemplo de El suicidio de Durkheim es importante en tanto se trata de una apropiación retrospectiva que la epidemiología hizo de esta obra" (Urquía, 2019, p.75). Sus aportes adquirirán relevancia en el período posterior a la Segunda Guerra Mundial, cuando la influencia de las ciencias sociales comience a permear el modelo médico. Hacia fines de la década de 1970, a nivel latinoamericano comienza a gestarse un movimiento académico crítico que propone la formulación de una nueva epidemiología que vaya más allá de lo metodológico y con base en la comprensión del proceso salud-enfermedad como fenómeno social y colectivo. Entre los principales participantes de este movimiento epidemiológico latinoamericano se encuentran Samuel Arias-Valencia, Naomar Almeida Filho, Ricardo Mendes-Gonçalves y Jaime Breilh.
El pensador portugués Boaventura de Sousa Santos ha acuñado el concepto "epistemología del sur" para caracterizar la producción de pensamiento en el sur del mundo donde "sur", más que un concepto geográfico, alude a un conocimiento contrahegemónico, que tiene su origen en contextos culturales y políticos propios, críticos de la epistemología occidental dominante (De Sousa Santos, 2009). Este tipo de movimiento epistemológico permitiría "no solo recuperar conocimientos suprimidos o marginalizados, sino también identificar las condiciones que tornen posible construir nuevos conocimientos de resistencia y de producción de alternativas al capitalismo y al colonialismo globales" (De Sousa Santos, 2009, p.12). Consideramos que, en el caso que analizamos, el modelo epidemiológico que desde el sur comienza a proponerse, asume estas características.

Para Naomar de Almeida Filho el contenido de la epidemiología crítica debe verificarse en la praxis histórica misma, y no en los modelos formales; sólo así, asumiendo intereses emancipatorios y enriquecedores, la teoría se vuelve ética y verdaderamente teoría (De Almeida, 1992). También Jaime Breilh postula que, a diferencia de la epidemiología moderna que asume la lógica de la perspectiva individual del liberalismo, la epidemiología crítica "no puede ser otra cosa que radicalmente emancipadora" (Breilh, 2013, p.14). Esto implica, además de la superación de los "enfoques epidemiológicos funcionalistas", la recuperación de la "unidad entre las necesidades populares y el quehacer científico" (Breilh; Granda, 1989, p.1121).

En términos generales, se describe para América Latina, durante el siglo XX, dos periodos en el desarrollo de la epidemiología psiquiátrica: una primera fase, a partir de los años treinta, donde los estudios se basan en entrevistas clínicas y cuestionarios no estandarizados sobre listas de síntomas, aun no validados; y una segunda, donde se definieron los estándares a seguir en la realización de las entrevistas y su aplicación en muestreos representativos de población. Es sin duda un camino trazado desde los centros de poder académico de Europa y Estados Unidos, que buscan la validación científica de la metodología clásica. Sin embargo, hacia mediados de siglo, desde la academia comenzará a estimularse la búsqueda de una epidemiología de los trastornos mentales acorde a la realidad de la Región. Postulamos que esta búsqueda asumirá el carácter de "movimiento" continental, del que Chile no sólo se hará parte, sino que será considerado por los mismos directores de la Oficina Sanitaria Panamericana como "país pionero" en su desarrollo y promoción (Mariátegui; Adís, 1970, p.12). 
El objetivo principal de la investigación que presentamos es analizar el surgimiento y desarrollo de este movimiento en Chile. Abordaremos el tema en tres segmentos. En el primero, trataremos los estudios epidemiológicos iniciales en Chile, su significado e importancia. El énfasis estará puesto en las conceptualizaciones que se proponen en torno a la epidemiología y a la salud mental. El segundo estará dedicado a la epidemiología del alcoholismo y su vinculación con el cambio de modelo asistencial. Finalmente abordaremos los cambios concretos en las políticas asistenciales. Postulamos como hipótesis central que, si bien originalmente los médicos buscaron, con la formulación de una epidemiología psiquiátrica, evidenciar una crisis social que se mantenía latente; en el corto plazo, esta búsqueda se convirtió en una oportunidad de transitar desde un modelo de asistencia asilar, centrado en la psiquiatría y la enfermedad, hacia otro centrado en la prevención y la salud pública.

Planteamos que este desarrollo significó, además, un cuestionamiento del modelo asilar imperante y una apertura hacia la psiquiatría social y la salud mental, lo que favoreció el desarrollo de políticas públicas vinculadas con las realidades particulares del país y su expansión a gran parte de Latinoamérica. Además del fracaso del modelo asilar, tanto la escasez de certezas científicas en torno al diagnóstico y terapéutica de los trastornos mentales, como la influencia de las ciencias sociales, se convirtieron en estímulos para desarrollar una epidemiología propia de los trastornos psiquiátricos, dejando de lado el modelo epidemiológico clásico.

\section{LOS PRIMEROS ESTUDIOS EPIDEMIOLÓGICOS EN CHILE}

Probablemente, debido al carácter social y cultural que ha tenido la ingesta de alcohol en Chile a lo largo de su historia, es que los primeros estudios epidemiológicos relacionados con la psiquiatría en este país se abocaron al estudio del alcoholismo (Fernández, 2005). Fueron además llevados a cabo por médicos psiquiatras, lo que se explica por la hegemonía que esta disciplina ha ejercido, en occidente en general, sobre la patología y la salud mental. Por esta misma importancia social del alcoholismo, es que Chile ha tenido, a nivel latinoamericano, una larga y reconocida trayectoria en este campo (Naveillan; Vargas, 1989).

El primer estudio epidemiológico sobre alcoholismo en el país fue realizado por el médico Luis Muñoz, utilizando el método indirecto, es decir, entrevistas aplicadas a terceros. Desde 1943, Muñoz venía desarrollando una línea de investigación dirigida a la búsqueda de "factores sociales" -así identificados por Mu- ñoz-, que explicaran ciertos aspectos del rendimiento pedagógico, del temperamento y del carácter moral de niños y adolescentes. Se trató de una serie de investigaciones reunidas como "Antecedentes para el estudio de la Higiene Mental del Escolar Primario", destinada a analizar las relaciones entre el nivel de vida y diversos aspectos psíquicos del escolar, implementado por enfermeras y visitadoras sociales. La aplicación de la encuesta a 1.000 familias de escolares de barrios obreros de Santiago evidenció "que el fracaso del aprendizaje fue más alto en los escolares que procedían de viviendas inferiores (conventillo y rancho) y en general, en los casos de promiscuidad de pieza y dormitorio" (Muñoz, 1955, p.212).

Como parte de esa investigación, Muñoz publica "El alcoholismo familiar y sus relaciones con el aprendizaje, el temperamento y el carácter en 1.000 escolares en Santiago", comprobando que, de un total de 534 familias de clase obrera, el 7,1\% de los padres eran alcohólicos, mientras que las madres lo eran en un $0,5 \%$ de los casos. Además, evidenció que un tercio de "los alumnos de hogares sostenidos por alcohólicos crónicos fracasaban en las más altas proporciones" (Muñoz, 1953, p.142). Algunos años antes, en 1950, en el Primer Congreso Mundial de Psiquiatría de París, había sostenido que "son causa importante de trastornos, de desadaptación, y de angustia, la desnutrición, la vivienda antihigiénica, el vestido deficiente, el alcoholismo de los padres o de los sostenedores del hogar, el abandono material y educacional de los niños y adolescentes" (Muñoz, 1955, p.218), adelantando de alguna manera los resultados de su investigación que había comenzado en 1943 y culminaría a mediados de la década de 1950, cuando la conceptualización de la salud mental se estaba consolidando ${ }^{2}$.

Luis Muñoz representa, desde nuestro punto de vista, una figura de transición entre la higiene y la salud mental, en el país. Si bien la conceptualización de sus investigaciones está planteada desde la higiene mental, la que define como "estudio y prevención de las causas que alteran la salud psíquica" considera que "la psiquiatría puede ser considerada como una ciencia social" en tanto "estudia los fenómenos o problemas sociales que alteran causalmente la salud mental de los individuos", introduciendo así conceptos como ciencia social y salud mental a un debate médico aun centrado en la higiene y el discurso nacionalista y de retórica social (Muñoz, 1950, p.9). En términos metodológicos, los trabajos de Muñoz no estaban destinados a medir prevalencia de alcoholismo, por lo que sus resultados son aproximativos. Pero más allá de la metodología, la importancia de sus investigaciones radica 
en la búsqueda de factores sociales que permitieran explicar el elevado consumo de alcohol en el país. Hasta 1955 todas las estimaciones de prevalencia de alcoholismo en el mundo se hacían por método indirecto. El más usado era la fórmula creada por el investigador húngaro Edwin Jellinek, a partir del número de muertos por cirrosis y de casos de alcohólicos con complicaciones.

El método indirecto logró superarse gracias a las investigaciones de salubristas chilenos, los que fueron asesorados por el mismo Jellinek en la década de 1950. En 1954, Juan Marconi realizó, a nivel mundial, el primer trabajo epidemiológico para medir la prevalencia del alcoholismo con método directo ${ }^{3}$. La investigación publicada al año siguiente como "A Survey of the Prevalence of Alcoholism Among the Adult Population of a suburb of Santiago" (Marconi, 1955), se basó en una muestra aleatoria de 787 familias de una comuna de Santiago a las que se le aplicó un instrumento basado en un estudio clínico previo de 100 alcohólicos. Se utilizaron como criterios la frecuencia de embriaguez y la duración y frecuencia de las crisis de ingestión. Las tasas de prevalencia encontradas, $5 \%$ de alcohólicos y $15 \%$ de bebedores excesivos, transformaron al alcoholismo en el "primer problema de Salud Pública del país, gracias a la aplicación del método epidemiológico" (Marconi, 1998, p.24).

Además de este último, el estudio de Marconi marcó otros dos grandes hitos en la historia de la salud mental en Chile. El método, además de aplicarse en todo el país, se aplicó en la mayoría de los países latinoamericanos, transformando a Chile en líder continental en este ámbito; y, no menos importante, marcó el inicio de la salud mental como disciplina médica en Chile. Es interesante constatar que al estudio de la prevalencia se sumaron aportes teóricos que marcaron la asistencia mental y las políticas públicas y que favorecieron, como veremos, una nueva epistemología en torno a la disciplina psiquiátrica. Uno de estos fue desarrollar conceptos operacionales para seis tipos psicopatológicos: alcoholismo, psicosis, demencia, oligofrenia, neurosis y epilepsia; lo que permitiría realizar estudios transculturales, comparando las investigaciones efectuadas en distintos países (Horwitz; Marconi, 1967).

A la investigación epidemiológica de Marconi siguieron otras que plantearon nuevos desafíos. En el período 1956-1957, el equipo liderado por el médico Ricardo Honorato, realizó un estudio similar, también tomando una muestra en una población obrera de Santiago. Sin embargo, la elevada tasa de prevalencia que arrojó el estudio (en comparación con el de Mar- coni y Muñoz) evidenció la necesidad de desarrollar una conceptualización precisa de la dependencia física como criterio diferencial entre el alcohólico y el bebedor excesivo simple. Entre 1957 y 1958, José Horwitz dirigió una investigación con método directo, que procuraba "establecer algunas relaciones ecológicas" respecto del alcoholismo y, realizar el "primer intento" de medir la prevalencia de epilepsia, de psicosis y de neurosis con una muestra más amplia y con definiciones más precisas acerca del alcoholismo y del beber excesivo, buscando salvar el problema que presentó el estudio de Honorato ${ }^{4}$. La investigación evidenció un $20 \%$ de prevalencia de trastornos mentales en mayores de 15 años, con los porcentajes más elevados para neurosis $(10 \%)$, bebedores excesivos $(7,7 \%)$ y alcoholismo (5,1\%) (Horwitz y otros, 1958). El estudio también mostró una asociación entre menor grado de instrucción, menor responsabilidad ocupacional y menor salario con una mayor proporción de alcohólicos y bebedores excesivos. Entre los aspectos socioculturales rescatados, se destacó la vinculación entre el hábito cultural de beber en exceso y el alcoholismo.

En resumen, estos primeros estudios epidemiológicos contribuyeron en varios sentidos. Los estudios pioneros de la década de 1940 de Luis Muñoz, sobre escolares de familias obreras, establecieron la complejidad del problema del alcoholismo y orientaron la psiquiatría hacia la medicina social y la salud pública. Es Muñoz el primero en considerar al alcoholismo como un "problema substrato", esto es, que, "al permanecer, hace movediza la plataforma de cualquiera acción que se pretenda hacer en salud, sea ésta relacionada con madre y niño, alimentación, atención médica, saneamiento, organización de la vida familiar o social, etc." (Muñoz, 1957, p.3); evidenciando así la influencia de los factores socioculturales en la salud mental. Los trabajos posteriores, especialmente de Marconi y Horwitz, en los que también participó Muñoz, situaron al alcoholismo como el problema de salud más importante de los chilenos desde mediados del siglo pasado. Es relevante el hecho de que tanto el alcoholismo como la epidemiología psiquiátrica se encontraban en un proceso de configuración; el alcoholismo no era aún conceptualizado como enfermedad y la epidemiología psiquiátrica no definía sus objetos de estudio ni su metodología. Esta sincronía determinará en gran medida el carácter que asuma cada uno de ellos.

\section{ALCOHOLISMO Y EPIDEMIOLOGÍA: HACIA UN NUEVO MODELO DE ASISTENCIA MENTAL}

Hacia la década de 1950, tanto las concepciones como las acciones de salud sobre el alcoholismo co- 
mienzan a cambiar en Latinoamérica. El término alcoholismo había sido ya acuñado a mediados del siglo XIX por el médico sueco Magnus Huss (Postel; Quétel, 1993). A partir de ese momento, y por diversas vertientes, se inicia un complejo proceso de medicalización del alcoholismo (Campos; Huertas, 1991). Sin embargo, fue en la década de 1940 cuando el investigador húngaro Edwin Jellinek desarrolló el concepto de "enfermedad del alcoholismo" (Jellinek, 1960) ${ }^{5}$. Su aporte fundamental fue definirlo a partir de la dependencia psicológica y especialmente física del alcohol; lo que favoreció el desarrollo de un nuevo enfoque y abrió "las puertas a las investigaciones epidemiológicas tan necesarias para conocer la ecología de una enfermedad de tan amplias repercusiones socioculturales" (Horwitz, 1967, p.12).

Con tal de generar conocimiento acerca de dicha problemática e iniciar los primeros estudios de prevalencia, en 1950 la Universidad de Chile creó el Instituto de Investigaciones sobre Alcoholismo, dirigido por Jorge Mardones, reconocido científico dedicado al tema. El Instituto se constituyó en dos niveles, investigación clínica y epidemiología. Una de sus primeras iniciativas fue solicitarle a Jellinek, director en ese momento del Instituto Internacional de Estudios sobre Problemas del Alcohol en Ginebra, que realizara asesorías sobre alcoholismo a médicos chilenos, las que comenzó en 1952. Posteriormente, en 1956, concretó una estadía de tres meses, donde trabajó con un grupo de técnicos del Servicio Nacional de Salud (S.N.S.) y de la Universidad de Chile. Una de las primeras actividades del grupo fue la organización de un Seminario donde se analizaron la experiencia chilena en el tema, las técnicas de educación sanitaria y el desarrollo de herramientas para calificar los hábitos de beber de la población. Los participantes elaboraron una encuesta de tipo antropológico, dirigida personalmente por Jellinek, el que tabuló los resultados obtenidos a lo largo de todo el país y los envió a Ginebra (Marconi, 1998).

En torno a estas primeras instancias comienzan a plantearse acciones concretas para enfrentar el problema del alcoholismo. En 1957, Mardones, Horwitz y Marconi, con el apoyo del S.N.S. elaboraron el primer Programa Nacional de Control y Prevención del Alcoholismo en hospitales generales, que dio pie a una serie de investigaciones e iniciativas sobre el tema. Una de ellas fue, ese mismo año, la organización en Chile del Primer Symposium sobre Alcoholismo y problemas del alcohol. Las exposiciones permitieron intuir tanto la elevada magnitud del problema, como la complejidad de los factores que incidían en su etiología. Es por esta misma complejidad, y por la falta de herramientas epidemiológicas, que gran parte de los investigadores aluden a la prevención y a la educación como factores ineludibles para enfrentar el asunto ${ }^{6}$.

En el evento, Guido Solari y Aníbal Varela relevaron la importancia de los factores socioculturales en la etiología del alcoholismo, a pesar de estar conscientes de la complejidad que comporta su estudio (Solari; Varela, 1957). Ante este panorama confuso, Luis Muñoz sostuvo que si bien no están capacitados para elaborar un programa preventivo integral basado exclusivamente en las causas; si es posible establecer "un camino promisor y efectivo de prevención" fundado en la "educación del niño, del adolescente y del adulto mismo" (Muñoz, 1957, p.4). Juan Marconi, insiste también en la necesidad de un programa preventivo, más allá de atacar el problema desde la etiología (Marconi, 1957, p.52) En la misma dirección, Miguel Cervantes y Francisco Mardones aluden al alcoholismo como un problema de salud pública y dado que en su génesis actúa " una constelación de factores", promueven un programa integral, que atienda al fomento de la salud y en el que deberían participar los ministerios de salud y de educación (Cervantes; Mardones, 1957, p.57).

Uno de los problemas capitales de la psiquiatría a lo largo de su desarrollo ha sido la falta de conceptualizaciones claras sobre los trastornos mentales, lo que representaba, en los inicios de la epidemiología, una gran dificultad para su despliegue. "Definiciones ambiguas, no posibles de traspasarse de un estudio a otro, observaciones ingenuas que paradójicamente se vuelven inmutables y dogmáticas", caracterizaban los estudios de ese tipo (Horwitz; Marconi, 1966, p.301). En esta época aun no existían nosologías consensuadas internacionalmente ni instrumentos validados. Ante esta ambigüedad, Horwitz y Marconi, proponen superar las definiciones puramente sintomáticas de desorden mental, las que tendrían escaso valor teórico y no serían aplicables en cualquier cultura, para tratar de producir conceptos estructurales y patogénicos, que serían los que tienen mayor valor como instrumentos transculturales.

Siguiendo esta misma línea investigativa publican, en 1967, "Estudios epidemiológicos y sociológicos acerca de la salud mental en Chile", donde insisten en la búsqueda de una epidemiología propia de los trastornos psiquiátricos incorporando aportes de la sociología a la discusión teórica y conceptual. Plantean que, al ser los trastornos psiquiátricos definidos de manera diferente, se hace necesario buscar "conocimientos comunes entre distintas culturas", es decir, apuntar hacia lo transcultural. Cuestionan también, indirectamente, 
aspectos importantes de la epidemiología moderna. Si en investigaciones previas establecían la necesidad de una conceptualización particular, aquí buscan que esa definición establecida se exprese "con criterios objetivos que permitan decidir a cada investigador, en cualquier caso particular, si el término se aplica o no a dicho caso" para, en un segundo momento, entender ese concepto enunciado "como una hipótesis de trabajo (...), de la cual se pueden extraer conclusiones lógicas susceptibles de ser sometidas a prueba con los enfoques clínico, epidemiológico y experimental" (Horwitz; Marconi, 1967, p.52). Apuntando a la idea de considerar a la epidemiología, más como una teoría que como una metodología.

Estas investigaciones representan un aporte significativo en, al menos, tres aspectos. En primer lugar, los autores esperaban que la población participante de estos estudios constituyera "el grupo inicial de aplicación de un programa demostrativo de psiquiatría de la comunidad, destinado a reorientar los programas nacionales de salud mental hacia una integración con los planes generales de salud pública" (Horwitz; Marconi, 1967, p.55). En segundo lugar, plantean la epidemiología como una teoría que se valida en la comunidad $y$, tercero, a través de las definiciones transculturales abren la puerta al relativismo cultural, a la antropología y las ciencias sociales, cambiando el enfoque desde la enfermedad psiquiátrica a la salud mental ${ }^{7}$. Nuevamente, desde las urgencias de las realidades de un país pobre y periférico, se proponen desafíos más allá de los modelos hegemónicos. Anticipando en parte las propuestas de Jaime Breilh sobre la epidemiología contrahegemónica.

Siguiendo la misma línea, hacia fines de la década de 1960, Marconi acusa al modelo vigente de sostenerse en una concepción vertical, etnocéntrica y autoritaria, donde la transmisión del conocimiento se realiza desde la cultura universitaria a un estrato "inferior" a través de la acción de los servicios de salud. Ante esta barrera cultural que se interpone, propone, para Latinoamérica, un modelo relativista cultural que coloque horizontalmente a la Universidad, a los servicios de salud y a las culturas de la comunidad, para enfrentar la salud mental como un objetivo común. El modelo debe ser respetuoso de todos los valores culturales y asumir la comunidad como el centro de las propuestas de salud mental. Según Marconi, en Chile coexisten tres modelos culturales médicos: el aborigen mapuche, el popular chileno y el europeo. Este último, hegemónico y etnocéntrico, solo ve las barreras económicas, pero no las culturales, por lo que es preciso corregir mediante programas de formación en antro- pología cultural que inculquen una actitud acorde con el principio de relativismo cultural (Marconi, 1970a).

Los estudios iniciados en Chile tuvieron repercusión en el resto del continente. El mismo grupo que participaba del Instituto de Investigaciones sobre Alcoholismo organizó, en 1957, en Viña del Mar, el Primer Seminario Latinoamericano sobre Alcoholismo. El evento, donde participaron 15 países, fue organizado en conjunto por el gobierno chileno y la Oficina Sanitaria Panamericana (O.P.S.), y se esperaba fuera una instancia para organizar programas de control en el continente, a través del intercambio de opiniones y experiencias sobre las definiciones de alcoholismo y su epidemiología. Ya en la inauguración, Abraham Horwitz, director de la O.P.S, posiciona a la epidemiología como una herramienta indispensable en el abordaje del alcoholismo. Sostiene que, más allá de la etiología o del conocimiento biológico, los avances epidemiológicos han permitido considerar a las enfermedades como "productos finales de una intrincada e inmensa relación entre los antecedentes ocultos con los términos de naturaleza o herencia y las variables sumergidas en las expresiones nutrición o ambiente" (Horwitz, 1960, p.4), contraponiendo el concepto epidemiológico clásico de "causa singular" por el de "nexo causal", instalando así una propuesta particular para la epidemiología de la salud mental donde se releva la prevención más allá del establecimiento de las causas.

A partir de esta experiencia, resulta interesante resaltar el carácter latinoamericanista que los salubristas buscaban imprimirle a la epidemiología del alcoholismo. Esto no solo se hace patente en el carácter continental de los eventos, sino en el interés por concretar una propuesta que tenga "la virtud de la trascendencia", para lo cual, al decir de Abraham Horwitz, se debe "conservar americana sin perder su sentido de universalidad" (Horwitz, 1960, p.6). Un ejemplo concreto es la réplica que se hizo en el distrito de Lanús, en Buenos Aires, del estudio de prevalencia de trastornos mentales en tres clases sociales, que el equipo de Laura Moya había realizado en Santiago en 1969 (Moya et al., 1969).

A fines de la década de 1960, muchos salubristas tenían la percepción de estar ante "una atmósfera en la cual podría desenvolverse la teoría y la práctica de la salud mental comunitaria, y una de sus raíces, la epidemiología psiquiátrica, en América latina" (Marconi, 1970a, p.161). Se esperaba que, a partir de la investigación epidemiológica, los distintos países generaran sus propios programas de control del alcoholismo. Es cierto que no podemos hablar de una crítica a la epi- 
demiología clásica, pero si podemos sostener que estamos frente a una propuesta de una epidemiología propia con los ejes puestos en la transculturalidad, el relativismo cultural y el comunitarismo. Según Marconi, "este movimiento, para nosotros tiene una trascendencia importante, nos ha unido, nos ha integrado culturalmente, (...) Nuestra tarea prioritaria es la integración latinoamericana en lo cultural, lo económico, y finalmente en lo político" (Marconi, 1982, p.425).

\section{ORIENTANDO LA PSIQUIATRÍA HACIA LA SALUD PÚBLICA}

En el plano nacional, si bien el desarrollo de los estudios epidemiológicos llevó a la formulación del Plan Nacional de Control del Alcoholismo en 1957, su continuidad y profundización comenzó a plantear nuevos desafíos tanto para la epidemiología como para la implementación de políticas públicas en torno a la salud mental. En este apartado analizaremos las nuevas problemáticas que comenzaron a surgir a partir de los estudios epidemiológicos y los cambios que generaron en las políticas públicas chilenas sobre el alcoholismo.

\section{Plan Nacional de Alcoholismo, 1957}

En 1957, en torno a las visitas de Jellinek al país y a la labor del Instituto de Investigaciones del Alcohol, se formuló un primer programa para enfrentar el asunto. En el Primer Symposium nacional sobre alcoholismo y problemas del alcohol realizado en Chile en 1957, Jellinek había criticado el que los "sobresalientes proyectos de investigación sobre alcoholismo" en Chile, se acompañaran de un "déficit total de programas de control, y de educación masiva de la población general" (Marconi, 1998, p.27). José Horwitz fue el motor del Programa, con la colaboración de Jorge Mardones, Luis Muñoz y Juan Marconi. Fue un programa básico que dejaba la educación sobre el problema del alcoholismo en manos de las escuelas primarias y la asistencia en los hospitales generales (Marconi, 1972, p.8).

\section{Programa nacional de control del Alcoholismo, 1964}

Luego de siete años de labor epidemiológica, en 1964, surgió el segundo Programa Nacional de control del Alcoholismo, elaborado por el S.N.S. El Programa, determinado en gran medida por los estudios epidemiológicos que lo precedieron, incorporaba definiciones precisas y operativas sobre el alcoholismo, planteaba como propósitos generales la disminución de la incidencia, de la prevalencia y del riesgo de morir por alcoholismo y sus complicaciones y proponía como ac- ciones generales el tratamiento, la rehabilitación y la prevención.

Este Programa, avanza respecto al anterior, al insertarse en el sistema de "hospital general" perteneciente a la red pública de asistencia de salud. Ahora bien, aun cuando proponía que los servicios locales adaptaran el Programa a sus realidades, este sistema se insertó en la organización jerárquica y centralizada del S.N.S., que concebía "la asistencia como tarea nacional, sin sectorización, incorporando técnicas psicoterapéuticas y psicofarmacológicas" (Marconi, 1998, p.18). Sin duda representó un aporte, pero también, dada la escasez de recursos económicos y humanos, hacía muy difícil su aplicación a nivel nacional. Tradicionalmente, además, la investigación científica de la medicina asistencial estaba orientada a la clínica y al tratamiento farmacológico, lo que impedía conocer la magnitud del problema del alcoholismo. Ante esta realidad, la emergencia de "la investigación epidemiológica, con su quemante denuncia de la magnitud del problema de Salud Mental, (...) actuó como motor de la siguiente etapa histórica" (Marconi, 1998, p.18). La evidencia de que un $25 \%$ de la población necesitaba atención mental, "rompe el esquema institucional de Hospitales, Consultorios, etc., para plantear el desafío de fondo: el traspaso del eje de los programas -asistencia, docencia e investigación- desde la estructura institucional a la estructura de masas, intracomunitaria, quedando las instituciones como elementos de apoyo y coordinación técnica" (Marconi, 1998, p.18).

\section{Primer Programa nacional de Salud Mental, 1966}

Además de los programas sobre alcoholismo, los estudios epidemiológicos sirvieron de impulso para que la sección de Salud Mental del S.N.S. y la Universidad de Chile formularan, durante 1966, por primera vez, un Programa Nacional de Salud Mental. Hubo que esperar 14 años, desde la creación del S.N.S. en 1952, para que se adoptara, con muy pocos recursos, una política nacional en este sentido. Sus autores, José Horwitz, Juan Marconi y Leonardo Muñoz, buscaban hacerse cargo del carácter comunitario que estaba desarrollando la psiquiatría en el país, para lo cual reorientaron los servicios desde los hospitales tradicionales a una cadena de instituciones ubicadas dentro de la comunidad, sectorizando esta última en 16 áreas a nivel nacional (Horwitz; Marconi, 1967).

Asimismo, descartaron el asilo y el hospital psiquiátrico "por su nula integración a la Salud Pública, su baja eficiencia, su acción iatrogénica en pacientes crónicos, su falta de sectorización, nula capacidad de crecimiento y alto costo" (Marconi, 1998, p.29). El 
modelo propuesto implicaba crear una red de instituciones: atención primaria en consultas periféricas; atención secundaria en consulta externa; urgencias psiquiátricas; psiquiatría de enlace; atención terciaria en hospitalización; rehabilitación en hogares y talleres protegidos. Todos los servicios serían atendidos por profesionales multidisciplinarios. Hasta el Golpe Militar de 1973, el Programa sólo había alcanzado a dar cobertura al $16 \%$ de la demanda nacional. A partir de ese momento su desarrollo fue "prácticamente nulo" (Marconi, 1998, p.29).

\section{De LA EPIDEMIOLOGÍA A LA ACCIÓN. MARCONI Y SU MODELO INTRACOMUNITARIO}

Aun siendo uno de sus autores, Marconi realiza una evaluación negativa del Programa Nacional de control del alcoholismo de 1966. Sus principales críticas, además de la falta de recursos, apuntaban a que se intentaba aplicar, en un país en vías de desarrollo y con población mayoritariamente obrera, un modelo que buscaba imitar lo realizado en países desarrollados, lo que describe como un "enfoque colonialista cultural" (Marconi, 1973, p.21). En base a estos cuestionamientos propone un nuevo modelo de salud mental, utilizando conceptos propios de las ciencias sociales, del marxismo y de la revolución cultural china, haciendo énfasis en las diferencias culturales y cuestionando las clasificaciones operacionales de los trastornos mentales ${ }^{8}$. Vale la pena detenerse en su visión, dado que esta derivará en el planteamiento de un modelo que, ya sea de manera explícita o simbólica, marcará el desarrollo de la salud mental de los chilenos hasta la actualidad (Araya; Leyton, 2017).

El modelo postulado por Marconi y sus colaboradores se plantea como un programa intracomunitario integral, basado en la atención primaria de salud y resultado de "la fusión de la eficiencia de la cultura popular para abordar el trastorno mental (obtener su propio objetivo al menor costo), con la eficacia terapéutica de la cultura y la medicina científicas" (Marconi, 1982, p.641). El único antecedente histórico del modelo comunitario urbano del sur de Santiago implementado por Marconi, a partir de 1968, fue el modelo comunitario rural de Abeokuta en Nigeria, en 1958. Uno de los aspectos más relevantes del programa integral intracomunitario es que pone en el centro de la política pública y de la epidemiología, tanto a la teoría epistemológica como a la acción social y política. Esto se traduce en la incorporación de conceptos hipotéticos que se ponen a prueba en la investigación misma. A partir de estas concepciones teóricas se desarrollan estrategias inéditas en el país para el desarrollo de políticas de salud mental: sectorización, trato no discrimina- torio para el paciente, democracia, descentralización, docencia masiva de líderes comunitarios, conceptos y metodologías de las ciencias sociales aplicadas, entre otros.

Marconi atribuía los problemas previos a la sociedad capitalista de clases, por lo que planteaba que era necesaria una revolución en salud mental (Marconi, 1973). Desde allí hace notar que no es lo mismo la ideología de la salud europea, de la salud obrera y de la aborigen, por lo que enmarca su análisis en los contextos antropológicos de cada uno de dichos grupos. Pero no solamente se queda en el plano especulativo, sino que también incorpora la manera en que los modelos culturales modifican o condicionan el enfermar. Por ejemplo, el beber excesivo en Chile, se originaría en la norma cultural masculina, aceptada a nivel nacional, de embriagarse después de la pubertad.

La instalación del programa intracomunitario comenzó en octubre de 1968, cuando Marconi debió hacerse cargo del Servicio de Salud Mental del área sur de Santiago, una zona pobre, con innumerables carencias en salud. Inmediatamente después de su llegada realizó un estudio en base a las consultas médicas del año anterior. Una medida que actualmente no representa ninguna novedad, pero que no era una práctica incorporada a la planificación de salud mental en esa época. El estudio permitió concentrar esfuerzos y recursos en las tres áreas con mayores consultas: alcoholismo $(38,9 \%)$, neurosis $(36,3 \%)$ y psiquiatría infantil (42\%). Por otra parte, la magnitud de consultas, especialmente por alcoholismo, evidenció que la aplicación del Programa Nacional era prácticamente imposible, dada la carencia de recursos. El éxito del programa de alcoholismo derivó en la implementación del programa de neurosis dirigido a mujeres adultas, en general esposas de los alcohólicos rehabilitados y, luego, en el de niños con privación sensorial.

El proceso anterior ha sido estudiado por la historiografía, pero es importante evidenciar otras experiencias que se llevaron a cabo fuera de Santiago. Entre 1971 y 1973, en paralelo con el programa institucional, se aplicó un programa intracomunitario en Antofagasta, una ciudad del norte chileno, con el supuesto de que este tipo de programas posibilitarían una mayor cobertura y rendimiento profesional, si es que se le asignaban los recursos suficientes, lo que permitiría superar, en el corto plazo, el viejo modelo asistencial (Minoletti; Pemjean, 1973). Los autores proponen, ante la "dramática" insuficiencia de recursos psiquiátricos en América latina, una reformulación de la asistencia mental basada en una nueva concepción de la 
enfermedad psiquiátrica, donde se tome en cuenta la "compleja y poderosa" influencia que en el paciente "ejerce su ambiente físico, social y cultural" y que se sume a la transformación del sistema de asilos en hospitales psiquiátricos especializados y a la "creación de otra serie de instituciones que tienden a un progresivo acercamiento de los profesionales a la sociedad misma" (Minoletti; Pemjean, 1973, p.436).

Ambos médicos eran parte del equipo de trabajo que había implementado Marconi en el área sur de Santiago, formado en gran parte por profesionales recién egresados o tesistas que participaban realizando sus investigaciones en epidemiología o en psiquiatría comunitaria. A partir de este trabajo en el norte, Minoletti y Pemjean proponen concretamente la creación de servicios de psiquiatría integrados en hospitales generales; consultorios periféricos; hospital diurno; talleres; hogares protegidos; granjas agrícolas; atención domiciliaria; clubes de ex enfermos y, probablemente lo más novedoso, una propuesta concreta sobre el desarrollo de la llamada "política de sector", considerada "un hito importante en este desarrollo..., en la cual un equipo multiprofesional, se responsabiliza integralmente de la salud mental de una población delimitada geográficamente" (Minoletti y Pemjean, 1973, p.436).

De manera similar a lo acontecido con Marconi al llegar al área sur de Santiago, cuando los autores llegan a Antofagasta y se encuentra con una grave escasez de recursos, deciden plantear para esa ciudad una experiencia en salud mental progresivamente intracomunitaria. Sin embargo, la realidad previa, de una tradición asistencial a nivel exclusivamente institucional, representó una importante limitación. Ante esta exigencia, se decidió trabajar simultáneamente en dos líneas complementarias. Por un lado, adecuar la institución y por otro, desarrollar un Programa Intracomunitario de Alcoholismo, para demostrar pedagógicamente una forma diferente de enfocar la salud mental. Se postuló como hipótesis el hecho de que, si un equipo de salud mental trabaja a tiempo parcial en la comunidad, obtendrá mejores resultados que continuando con la asistencia tradicional.

En 1973, luego del Golpe Militar y de la desarticulación de los programas intracomunitarios y de las políticas de salud mental en general, Marconi, movido por el constante acoso de los militares, abandonó el país y se dirigió a Córdoba. Allí influyó a otros colegas (Marconi, 1974; Marconi; Saforcada, 1974) que implementaron los programas comunitarios que había iniciado en Chile y que se constituyeron, según sus palabras, en "un verdadero laboratorio social para poner a prueba el cambio desde el modelo asilo-hospital psiquiátrico al programa integral de salud mental" (Marconi, 1976, p.117). Cuando vuelve a Chile, en 1976, aún mantenía la convicción de que los programas podían mantenerse. De ahí que su propuesta de ese año para Latinoamérica mantenga lo sustancial de los programas iniciales, en términos teóricos, pero advirtiendo sobre una realidad mucho más adversa. Sus palabras suenan ahora más moderadas que al inicio de la década. Si el diagnóstico previo indicaba que, si bien la solución correcta sería "programar como solución única la construcción de unidades de salud mental a nivel nacional", la realidad de ese momento mostraba la necesidad de mantener los programas de construcción de hospitales psiquiátricos o asilos para crónicos (Marconi, 1976, p.116).

Como alternativa a la constitución de unidades de salud mental a nivel nacional, propone la implementación de programas integrales intermedios de "bajo costo, de acelerada difusión, de dinámica interna alta, que utilice recursos locales" y que, a mediano plazo, cubran el vacío "históricamente determinado por nuestro subdesarrollado industrial" (Marconi, 1976, p.116) La fase final del programa integral sería la instalación completa de la unidad de salud mental, con su compleja y costosa cadena de servicios. De esta manera, se invierte el sentido de programación clásico. Ya no resultaría necesario el requisito de construir un centro de salud mental en el hospital base de un área, sino que se podría comenzar con "los recursos locales más periféricos, el hogar de un alcohólico o un neurótico recuperados" (Marconi, 1976, p.117). El énfasis fundamental no está en los recursos materiales, como en los programas anteriores, sino en los recursos humanos, y en particular, en los líderes comunitarios que trabajan voluntariamente, y obtienen rendimiento máximo con costo mínimo.

Sin embargo, en la práctica, los programas no tuvieron continuidad. Hacia la década de 1980, la gravedad del problema del alcoholismo persistía en Chile, a pesar de ser un país pionero a nivel mundial en investigación epidemiológica y asistencia. Entre 1973 y 1990, prácticamente no hubo ningún estudio epidemiológico, salvo una réplica, 25 años después, del estudio realizado por el equipo de Horwitz sobre prevalencia del alcoholismo en Santiago (Naveillan; Vargas, 1989). Según Marconi, "el contexto nacional que sustentaba estos avances era, entre muchos otros temas, la continuidad democrática del país, (...) el crecimiento notable de la Universidad de Chile, con sedes en todo el país; (...) la creación, en 1952, del Servicio Nacional de Salud" (Marconi, 1998, p.22). Lo que vino a continuación fue la "pérdida abrupta de la inserción 
comunitaria" desencadenada por la Dictadura (Marconi, 1998, p.38). A este período, de 1973 a 1990, Marconi lo llama "de involución", porque se paralizaron los programas, hubo "nula asignación de recursos a Salud Mental" y se agregaron problemas nuevos, producto de la violencia institucionalizada (Marconi, 1998, p.41). Una realidad no solo nacional sino continental. Para el psiquiatra chileno, "el rasgo más relevante de la psiquiatría latinoamericana desde 1950 a la fecha es su carácter de fracturada, fiel reflejo de una historia constitucional también quebrada, repetidamente, por golpes militares" (Marconi, 1982, p.423).

Nos reencontramos, hacia el final, con la figura paradojal usada por Marconi para caracterizar el devenir de la salud mental Latinoamericana: aquella de que los salubristas mentales viven "recordando el futuro". Porque, desde 1973, en el caso chileno, lo único que "permanece sólido e inconmovible es el hospital psiquiátrico" y, "todo lo que intenta nacer, ya sea una simple comunidad terapéutica dentro de ese modelo, ya un servicio de psiquiatría moderno, en un hospital general, o un programa comunitario en un área de salud es, con demasiada frecuencia, quebrado, reprimido, privado de recursos o asfixiado de mil maneras por el dictador de turno". Mientras que, a nivel externo, "el factor más poderoso en el devenir histórico (...), es la dominación norteamericana, que lleva a nuestros países con un sistema de capitalismo dependiente, a bailar al son que nos tocan" (Marconi, 1982, p.423).

\section{CONCLUSIONES}

El desarrollo de la epidemiología de los trastornos mentales en Chile está estrechamente ligado al alcoholismo y a su configuración como enfermedad primero, y como problema de salud mental posteriormente. Desde los primeros estudios en la década de 1940 se asume que el alcoholismo debe abordarse como una enfermedad social multifactorial, donde además de la prevalencia, es necesario investigar los factores sociales y ambientales que lo determinan. En esa línea se insertaron los trabajos de Luis Muñoz, quien, como médico y pedagogo, buscó vincular la ingesta de alcohol de los padres con el rendimiento escolar de los hijos. Cuando aún no se desarrollaba un método directo para estudiar la prevalencia del alcoholismo, su propuesta fue avanzar hacia la prevención desde la educación. Estos primeros estudios denotan no sólo un interés descriptivo, sino que muestran, a nuestro entender, una intención clara por teorizar en torno a la epidemiología del alcoholismo.
Entre las décadas de 1930 y 1950, teniendo como eje el Hospital Psiquiátrico de Santiago y la cátedra de psiquiatría de la Universidad de Chile, en el área norte de Santiago, los psiquiatras chilenos habían afianzado el carácter biologicista de la disciplina. Sin embargo, desde la década de 1950, con el apoyo de la Universidad de Chile, la O.P.S. y el Instituto de Investigaciones sobre Alcoholismo, comienzan a multiplicarse los estudios epidemiológicos sobre prevalencia, los que permitieron situar al alcoholismo como el principal problema de salud pública, estimular cambios en la concepción psiquiátrica de los trastornos mentales y desarrollar un enfoque de salud mental y salud pública. Las características geográficas, la escasez de recursos, las diferencias étnicas y culturales y el momento político y social que vivía Chile, en las décadas de 1960 y 1970, facilitaron el desarrollo de una epidemiología crítica, anticipando el surgimiento de este movimiento en Latinoamérica en la década de 1980, en el sentido que se buscaba producir conocimiento teórico en torno a las particularidades locales y no sólo una metodología objetiva para medir prevalencia.

Chile se convirtió en un polo científico y social en el continente, desde donde se propusieron conceptualizaciones en torno a los trastornos mentales y políticas de salud mental comunitaria en torno al alcoholismo. Las diversas instancias académicas generadas en América latina en torno a la epidemiología de la salud mental y del alcoholismo, son muestras de lo mismo. El surgimiento de la epidemiología del alcoholismo en Chile llevó a la formulación de los primeros programas de control con enfoque de salud pública, siguiendo los modelos sanitarios exitosos implementados luego de la creación del Servicio Nacional de Salud en 1952. Sin embargo, la continuidad de los mismos estudios llevó a cuestionar tanto su conceptualización como su impacto. La maduración de la epidemiología del alcoholismo, su despliegue a otras zonas del país y la concientización política de los principales actores respecto a la necesidad de un cambio, desde el enfoque fundamentalmente biomédico a otro científico-social, llevó a proponer e implementar programas de salud mental integrales e intracomunitarios. Las evaluaciones iniciales resultaron alentadoras. Sin embargo, como ya hemos dicho, la violenta disrupción del tejido social y político provocado por la dictadura cívico militar, desarticuló los programas y las políticas de salud mental.

El desarrollo de una epidemiología crítica, de esas características, está muy ligado a lo político, y, fue posible, en gran parte, por el grado de politización que había alcanzado el país y las instituciones universitarias. 
La desarticulación violenta de los procesos políticos y sociales a partir de 1973, por la Dictadura cívico-militar, implicó necesariamente la interrupción abrupta del desarrollo de una escuela epidemiológica crítica, que se estaba convirtiendo en un movimiento a nivel latinoamericano. Así como empezábamos este artículo con la cita de Marconi sobre la psiquiatría latinoamericana, hemos dejado para terminar el epígrafe de Jaime Breilh que introduce esta investigación. Hoy en Chile, más de 60 años después de la implementación del Primer Programa de Control, el alcoholismo continúa siendo uno de los principales problemas de salud mental. Sin duda que el modelo de sociedad neoliberal

\section{NOTAS AL FINAL}

1 El suicidio es en Chile la principal causa de muerte en el grupo de 20 a 44 años. Según el estudio Costos Económicos y Sociales del Consumo de Alcohol en Chile 2018, uno de cada tres escolares de entre 13 y 18 años dice haber consumido alcohol durante el último mes, y de ellos, el 61,7\% declara haberse embriagado.

2 En 1966, Laura Moya evidenció que el 17,4\% de los escolares de un barrio obrero de Santiago presentaban problemas de salud mental. El estudio establecía una continuidad con las investigaciones de Muñoz, que fue interrumpida por el Golpe Militar de 1973 (la misma Dra. Moya fue exonerada y perseguida por la Dictadura) y retomada en la década de 1990, con escaso presupuesto y proyección (Moya, 1966).

3 Juan Marconi, nació en Valparaíso en 1924 y murió en el sur chileno en 2005. Psiquiatra y profesor universitario, se interesó tempranamente por la epidemiología de los trastornos mentales. En la década de 1960 desarrolló una teoría estructural de la psicosis que se mantuvo vigente en Chile hasta fines del siglo pasado. Participó en la Reforma Universitaria y desarrolló definiciones y enfoques clínicos sobre el alcoholismo inveterado y el intermitente. Probablemente su principal influencia se vincula con los programas intracomunitarios de salud mental que comienza a implementar desde fines de la década de 1960 en el área sur de Santiago.

4 Como indicador de alcoholismo se consideró la incapacidad

\section{BIBLIOGRAFÍA}

Araya, Claudia; Leyton, César (2017), “La 'vía chilena' a la psiquiatría social. Propuestas y debates en torno al cambio de modelo de asistencia mental, 1968-1973". En: Huertas, Rafael (coord.), Políticas de salud mental y cambio social en América Latina, Madrid, Catarata, pp. 56-82.

Araya, Claudia (2018), La locura es nuestra: profesionalización de la psiquiatría en Chile. Saberes y prácticas, 1826-1949, Rosario, Prohistoria.

Barradas Barata, Rita (1998), "Epidemiologia e saber científico", Revista Brasileña de Epidemiología, 1(1), pp. 14-27. impuesto por la Dictadura y administrado y ampliado por los gobiernos posteriores, es la respuesta principal a esta situación. Actualmente nos encontramos en un proceso de levantamiento social, articulado principalmente en torno al rechazo al modelo neoliberal, responsable de la desigualdad abismante que caracteriza a la sociedad chilena, refrendada por el Informe Desiguales, del Programa de Naciones Unidas para el Desarrollo (PNUD) de 2017. Por lo mismo, estamos ante una oportunidad histórica para, al decir de Breilh, la "construcción de una politicidad" que, en el caso que nos ocupa, conlleve al desarrollo de una epidemiología ética que vuelva a situar al individuo en la comunidad.

de detenerse durante la ingestión de alcohol. Se usaron también indicadores suplementarios como la frecuencia de embriaguez, la cantidad de crisis de ingestión de alcohol en un año y algunos síntomas de privación.

5 La dedicatoria de la obra clásica de Jellinek al médico salubrista chileno Jorge Mardones es un reconocimiento a la importancia de las investigaciones chilenas.

6 Un segundo Symposium sobre Alcoholismo se realizó en 1964. Desde ahí surgió la propuesta de ampliar el Programa Nacional de Control del Alcoholismo al resto de los trastornos mentales, lo que se concretó en 1966.

7 En otros estudios, Horwitz y Marconi analizaron la validez de definiciones transculturales para desórdenes mentales. Uno de ellos fue la realización de una investigación epidemiológica de campo en población chilena y mapuche del centro de Chile. Para el estudio de los segundos, analizaron las historias clínicas de mapuches egresados del Hospital Psiquiátrico de Santiago, entre 1940 y 1963. Los resultados mostraron diferencias entre los dos grupos en "psicosis asociada a deficiencia mental y en psicosis reactivas" (Horwitz; Marconi, 1966, p.305).

8 Victoria Toro, enfermera y esposa de Marconi, relata que mientras trabajaba en la formulación de los programas intracomunitarios de salud mental, Marconi, se ausentó durante una semana de su trabajo para estudiar todo lo referente a la Revolución Cultural China. Entrevista a Victoria Toro, Santiago, 16 de junio de 2018.

Breilh, Jaime; Granda, Edmundo (1989), “Epidemiología y contrahegemonía”, Social Science \& Medicine, 28(11), pp. 11211127.

Breilh, Jaime (2013), Epidemiología crítica: Ciencia emancipadora e interculturalidad, Buenos Aires, Lugar.

Campos, Ricardo; Huertas, Rafael (1991), “El alcoholismo como enfermedad social en la España de la Restauración: problemas de definición", Dynamis, 11, pp. 263-286.

Castel, Françoise; Castel, Robert; Lovell, Anne (1980), La sociedad psiquiátrica avanzada. El modelo norteamericano, Barcelona, Anagrama. 
Cervantes, Miguel; Mardones, Francisco (1957), "Bases para un programa de tratamiento y prevención del alcoholismo". En: Symposium sobre alcoholismo y problemas del alcohol, Santiago, Servicio Nacional de Salud, Imprenta Central de Talleres, pp. 57-62.

De Almeida, Naomar (1992), Epidemiología sin números. Una introducción crítica a la ciencia epidemiológica, Organización Panamericana de la Salud.

De Sousa Santos, Boaventura (2009), Una epistemología del Sur: la reinvención del conocimiento y la emancipación social, México, Siglo XXI-Clacso.

Durkheim, Emile [1897] (2013), El suicidio, México, Colofón.

Fernández, Marco (2005), Historia social del alcoholismo en Chile, 1870-1930: políticas, prácticas, representaciones, Santiago, Tesis doctorado en Historia, Pontificia Universidad Católica de Chile.

Ferrero, Andrea (2000), "El concepto de epidemiología psiquiátrica. Un recorrido histórico", Acta Psiquiátrica y Psicológica de América Latina, 46(1), pp. 75-79.

Foucault, Michel (2009), Seguridad, territorio y población: curso en el Collège de France, 1977-1978, Buenos Aires, Fondo de Cultura Económica.

Horwitz, Abraham (1959), "Mensaje del Dr. Abraham Horwitz", Boletín del Servicio Nacional de Salud, 5(2), pp. 164-165.

Horwitz, Abraham (1960), "Discurso inaugural”. En: Primer Seminario Latinoamericano sobre Alcoholismo, Viña del Mar, 21-26 de noviembre de 1960, Servicio Nacional de Salud, Oficina Sanitaria Panamericana, pp. 3-6.

Horwitz, Abraham (1963), "Propósitos y proyecciones del Seminario Latinoamericano de Salud Mental". En: Primer Seminario Latinoamericano de Salud Mental, Cuernavaca, 23 de noviembre-3 de diciembre de 1962, Publicaciones Científicas No.81, Organización Panamericana de la Salud, Organización Mundial de la Salud, pp. 3-4.

Horwitz, Abraham (1964), "Introducción". En: Segundo Seminario Latinoamericano de Salud Mental, Buenos Aires, 8-14 de septiembre de 1963, Publicaciones Científicas No.99, Organización Panamericana de la Salud, Organización Mundial de la Salud, pp. v-vii.

Horwitz, José; Muñoz, Luis; Álvarez, Madeline; Becerra, María (1958), "Investigaciones epidemiológicas acerca de morbilidad mental en Chile", Revista del Servicio Nacional de Salud, 3(3-4), pp. 277-309.

Horwitz, José (1961), "El uso del método epidemiológico en psiquiatría", Boletín de la Oficina Sanitaria Panamericana, 50(6), pp. 505-512.

Horwitz, José (1963), "Epidemiología de los problemas de salud mental". En: Primer Seminario Latinoamericano de Salud Mental, Cuernavaca, 23 de noviembre-3 de diciembre de 1962, Publicaciones Científicas No.81, Organización Panamericana de la Salud, Organización Mundial de la Salud, pp. 29-37.

Horwitz, José (1964), "La importancia del problema de la salud mental. Contribución al estudio epidemiológico de los desórdenes mentales". En: Segundo Seminario Latinoamericano de Salud Mental, Buenos Aires, 8-14 de septiembre de 1963 Publicaciones Científicas No.99, Organización Panamericana de la Salud, Organización Mundial de la Salud, pp. 16-24.

Horwitz, José (1965), "La obra de Jellinek y su importancia para el conocimiento del alcoholismo en Chile", Acta Psiquiátrica y Psicológica de América Latina, 11(1), pp. 81-85.

Horwitz, José, Marconi, Juan (1966), “El problema de las definiciones en el campo de la salud mental. Definiciones aplicables en estudios epidemiológicos", Boletín de la Oficina Sanitaria Panamericana, 60(4), pp. 300-309.

Horwitz, José (1967), “Introducción”. En: Horwitz, José; Marconi, Juan; Adís Castro, Gonzalo, (eds.), Bases para una epidemiología del alcoholismo en América Latina, Buenos Aires, Acta Fondo para la salud mental, pp.11-16.

Horwitz, José; Marconi, Juan; Adís, Gonzalo (1967), Bases para una epidemiología del alcoholismo en América Latina, Buenos Aires, Acta Fondo para la salud mental.

Horwitz, José; Marconi, Juan (1967), “Estudios epidemiológicos y sociológicos acerca de la salud mental en Chile. Comunicación preliminar", Acta Psiquiátrica y Psicológica de América Latina, 13(1), pp. 52-58.

Jellinek, Elvin (1960), The disease concept of alcoholism, New Haven, Hillhouse Press.

Klappenbach, Hugo (1989), "Higiene mental en las primeras décadas del siglo. Principales lineamientos y fundamentos ideológicos", Anuario de Investigaciones, 1, 189-195.

Marconi, Juan (1955), "A survey on the prevalence of alcoholism among the adult population of a suburb of Santiago", Quart. J. of Stud. Alc., 16, pp. 438-446.

Marconi, Juan (1957), "Tratamiento y Prevención”. En: Symposium sobre alcoholismo y problemas del alcohol, Santiago, Servicio Nacional de Salud, Imprenta Central de Talleres, pp. 43-55.

Marconi, Juan; Muñoz, Leonardo (1970), "Visión general de la investigación epidemiológica en salud mental en Chile". En: Mariátegui, Javier; Adís, Gonzalo (eds.), Estudios sobre epidemiología psiquiátrica en América Latina, Buenos Aires, Acta. Fondo para la salud mental, pp. 115-125.

Marconi, Juan (1970a), "Esbozo de modelos de valor epidemiológico para la investigación y la acción en América latina". En: Mariátegui, Javier; Adís, Gonzalo (eds.), Estudios sobre epidemiología psiquiátrica en América Latina, Buenos Aires, Acta Fondo para la salud mental, pp. 161-179.

Marconi, Juan (1970b), "Una teoría psicopatológica estructural de la psicosis", Acta Psiquiátrica y Psicológica de América Latina, 16(1), pp. 15-32.

Marconi, Juan (1971), “Asistencia psiquiátrica intracomunitaria en el área sur de Santiago. Bases teóricas y operativas para su implementación (1968-1970)", Acta Psiquiátrica y Psicológica de América Latina, 17(3), pp. 255-264.

Marconi, Juan (1972), "Asistencia psiquiátrica intracomunitaria en el área sur de Santiago. Bases teóricas y operativas para su implementación (1968-1970)", Revista Chilena de Neuropsiquiatría, 11(2), pp. 3-12. 
Marconi, Juan (1973), “La revolución cultural chilena en programas de salud mental", Acta Psiquiátrica y Psicológica de América Latina, 19(1), pp. 17-33.

Marconi, Juan (1974), “Diseño de un programa integral de salud mental para la ciudad de Córdoba", Acta Psiquiátrica y Psicológica de América Latina, 20(3), pp. 240-246.

Marconi, Juan; Saforcada, Enrique (1974), “Formación de personal para un programa integral de salud mental en Córdoba", Acta Psiquiátrica y Psicológica de América Latina, 20(6), pp. 403-410.

Marconi, Juan (1976), "Política de salud mental en América Latina", Acta Psiquiátrica y Psicológica de América Latina, 22(4), pp. $112-120$.

Marconi, Juan (1982), “La psiquiatría y la salud pública: un modelo de salud mental". En: Gomberoff, Mario; Jiménez, Juan Pablo (eds.), Psiquiatría, Santiago, Medcom, pp. 631-649.

Marconi, Juan (1990), "Voces de la psiquiatría latinoamericana: entrevista a Juan Marconi". En: Alarcón, Renato, Identidad de la psiquiatría latinoamericana. Voces y exploraciones en torno a una ciencia solidaria, México, Siglo veintiuno editores, pp. 420-435.

Marconi, Juan (1998), Del asilo a la comunidad: 50 años de psiquiatría chilena, 1948-1998. Un testimonio personal, Inédito.

Mariátegui, Javier; Adís, Gonzalo (1970), Estudios sobre epidemiología psiquiátrica en América Latina, Buenos Aires, Acta Fondo para la salud mental.

Mendive, Susana (2004), “Entrevista al Dr. Juan Marconi, creador de la psiquiatría intracomunitaria. Reflexiones acerca de su legado para la psicología comunitaria chilena", Psikhe, 13(2), pp. 187-199.

Minoletti, Alberto; Pemjean, Alfredo (1973), "Salud mental en el norte de Chile: Un desafío teórico y operacional", Acta Psiquiátrica y Psicológica de América Latina, 19(4), pp. 434444.

Minoletti, Alberto (2006), "Semblanza Juan Marconi Tassara”, Revista Chilena de Neuropsiquiatría, 44(1), pp. 61-62.

Moya, Laura (1966), "Problemas de salud mental en una escuela primaria de Santiago", Acta Psiquiátrica y Psicológica de América Latina, 12(1), pp. 63-68.

Moya, Laura; Marconi, Juan; Horwitz, Jorge; Muñoz, Luis; Bahamondes, Andrés; Saint-Jean, Héliettè (1969), "Estudio de prevalencia de desórdenes mentales en el Área Norte de
Santiago de Chile. Comparación de poblaciones de tres niveles socioeconómicos", Acta Psiquiátrica y Psicológica de América Latina, 15(2), pp. 137-148.

Muñoz, Luis [1950] (1962), “Comunicación de Luis Custodio Muñoz al Primer Congreso Mundial de Psiquiatría". En: Introducción a la higiene mental, Santiago, Servicio Nacional de Salud.

Muñoz, Luis (1953), "El alcoholismo familiar y sus relaciones con el aprendizaje, el temperamento y el carácter en 1.000 escolares de Santiago". En: Congreso Internacional de Neuropsiquiatría, IX Jornada Nacional, 9 al 15 de diciembre de 1952, Tomo II, Santiago de Chile, pp. 141-143.

Muñoz, Luis [1955] (2011), “¿Qué es la psiquiatría social?”. En: Villaseñor, Sergio; Rojas, Carlos; Garrabé de Lara, Jean (eds.), Antología de textos clásicos de la psiquiatría latinoamericana, Guadalajara, Gladet, pp.211-213.

Muñoz, Luis (1957), "El problema del alcoholismo". En: Symposium sobre alcoholismo y problemas del alcohol, Santiago, Servicio Nacional de Salud, Imprenta Central de Talleres, pp. 3-4.

Naveillan, Pedro; Vargas, Sergio (1989), "Prevalencia del alcoholismo durante tres décadas en Chile (1952-1982)", Revista de Saúde Pública, 23(2), pp. 128-135.

Oficina Sanitaria Panamericana (1960), Seminario Latinoamericano sobre alcoholismo. Organizado por la Oficina Sanitaria Panamericana, Oficina Regional de la Organización Mundial de la salud, con la colaboración del Servicio Nacional de Salud y el auspicio de la Universidad de Chile y del Colegio Médico de Chile, Viña del Mar, Chile, 21-26 de noviembre de 1960.

Postel, Jacques; Quétel, Claude (eds.) (1993), Historia de la psiquiatría, México, Fondo de Cultura Económica.

Solari, Guido; Varela, Aníbal (1957), "Factores etiológicos del alcoholismo". En: Symposium sobre alcoholismo y problemas del alcohol, Santiago, Servicio Nacional de Salud, Imprenta Central de Talleres, pp. 29-42.

Urquía, Marcelo (2019), Teorías dominantes y alternativas en epidemiología, Lanús, Universidad Nacional de Lanús.

Williams, Paul; Wilkinson, Greg; Rawnsley, Kenneth (eds.) (2019), The Scope of Epidemiologycal Psychiatry. Essays in honour of Michael Sheperd, New York, Routledge. 